\title{
La argumentación como constituyente del pensamiento crítico en niños ${ }^{*}$
}

\author{
Oscar Eugenio Tamayo Alzate**
}

\begin{abstract}
RESUMEN
Recibido: 8 de septiembre de 2011

Revisado: 28 de octubre de 2011 Aprobado: 24 de noviembre de 2011

El informe de investigación que presentamos a continuación hace parte de un proyecto de investigación que estudia el pensamiento crítico en niños desde tres categorías: solución de problemas, argumentación y metacognición. En estas páginas solo nos referiremos a los procesos argumentativos realizados por niños de $4^{\circ}$ y $5^{\underline{o}}$ grado de educación básica primaria, para lo cual seguimos un diseño metodológico mixto. Para la intervención didáctica en función de desarrollar pensamiento crítico en los estudiantes se diseñaron diez actividades, las cuales fueron aplicadas a 2.200 niños de 56 instituciones educativas públicas. Los análisis cualitativos y cuantitativos fueron realizados con 220 niños. Los principales resultados describen las estructuras argumentativas empleadas por los niños durante las diferentes actividades de aula presentadas. Concluimos con algunas recomendaciones desde la didáctica de las ciencias orientadas a cualificar los procesos y productos argumentativos.
\end{abstract}

\section{Palabras clave}

pensamiento crítico, argumentación, didáctica.

\footnotetext{
* Este artículo de investigación constituye uno de los productos obtenidos en el marco del proyecto: Formación de pensamiento crítico en niños mediante la Enseñanza de las Ciencias. Colciencias. Código 1127-452-21366.

** Profesor, Universidad de Caldas y Universidad Autónoma de Manizales. Correo electrónico: oscar.tamayo@ucaldas.edu.co
} 


\title{
Argumentation as a critical thinking constituent in children
}

\author{
Oscar Eugenio Tamayo Alzate
}

\begin{abstract}
This research report is part of a research project that studies critical thinking in children in three categories: problem solving, reasoning and metacognition. In the following pages we will refer only to reasoning processes carried out by children in $4^{\text {th }}$ and $5^{\text {th }}$ grade of elementary school. In order to reach our goal, we followed a mixed methodological design. For the educational intervention which aimed to develop critical thinking in students, ten activities were designed and implemented with 2200 children from 56 public educational institutions. The qualitative and quantitative analyses were conducted with 220 children. The main results describe the argumentative structures used by the children while undertaking the different classroom activities presented. We conclude with some recommendations from the perspective of science didactics designed to qualify reasoning processes and products.
\end{abstract} Aprobado: 24 de noviembre de 2011

\section{Keywords}

Critical thinking, argumentation, science education. 


\section{INTRODUCCIÓN}

En el estudio del pensamiento crítico se pueden asumir posturas filosóficas, sociológicas y psicológicas dentro de las cuales, sin lugar a dudas, se han realizado y se continuarán haciendo desarrollos importantes. No obstante lo anterior, los resultados de investigación que aquí presentamos tienen sus raíces en la educación; nos interesa el estudio del pensamiento crítico en relación con los procesos de enseñanza y aprendizaje de las ciencias, procesos en los cuales incorporamos aportes valiosos de diferentes campos disciplinares.

En el campo de la educación, afirmamos que el objeto de estudio central y específico de la didáctica de las ciencias es la formación del pensamiento crítico en los estudiantes. Hoy las didácticas no tienen como único propósito la enseñanza, el aprendizaje, el diseño de ambienes educativos o la evaluación; su propósito central es aportar a la formación de ciudadanos comprometidos tanto con el desarrollo individual como social. Así mismo, en la actualidad el actuar de los maestros en las aulas de clase no se circunscribe a la enseñanza de conceptos o de teorías, propósito central de la didáctica de las ciencias de las décadas pasadas.

Priorizar la formación del pensamiento crítico en los estudiantes mediante la didáctica de las ciencias implica transformar las prácticas de los maestros. No será a partir de las estrategias de enseñanza tradicionales como logremos avanzar en la constitución de pensamiento crítico; es indispensable el uso de principios teóricos y metodológicos coherentes con este propósito. En las páginas que presentamos a continuación haremos, inicialmente, un breve recorrido por diferentes ideas acerca del pensamiento crítico y, posteriormente, nos referiremos de manera específica a algunos resultados de investigación en una de las dimensiones que más capta la atención en estos momentos en la didáctica de las ciencias naturales: la argumentación como constituyente del pensamiento crítico.

Bruner, en su texto La importancia de la educación (1987), ubica las posibles dificultades del hombre moderno para comprender las matemáticas y las ciencias, no en la falta de capacidades, sino en que no logramos encontrar un camino adecuado y eficiente para su enseñanza. La evidencia que permite asegurar que los estudiantes de ciencias no aprehenden realmente el conocimiento que se les enseña se manifiesta en forma clara en su incapacidad para utilizar los conocimientos en la explicación de fenómenos cotidianos.

- Los alumnos no aprenden los conceptos fundamentales de las ciencias, sino una caricatura de ellos.

- No entienden el funcionamiento de las máquinas que usan a diario ni son capaces de aplicar los principios de su funcionamiento.

- La enseñanza en la escuela, en lugar de ser divertida e interesante, es frustrante para el estudiante.

Dentro de las principales causas que explican las observaciones anteriores encontramos el modelo utilizado para la enseñanza de las ciencias, en el cual no se ha reconocido el dinamismo de las ciencias, su larga historia y, menos aún, su desarrollo histórico como un elemento importante para su comprensión y para la orientación de su 
enseñanza. Es un modelo en el cual se considera al alumno como el sujeto que aprende, desprovisto de historia. Esto ha conducido al ejercicio de una enseñanza agenética en la que trata de enseñarse todo en poco tiempo, desconociéndose así la experiencia del estudiante. De igual manera, la enseñanza tradicional de las ciencias pretende explicar su estructura lógica actual, sin hacer evidente el engranaje conceptual que la hace posible; esto dificulta su aprendizaje. Lograr avanzar en la formación de pensamiento crítico en los estudiantes necesariamente se articula con los modelos pedagógicos y didácticos desde los cuales pensamos y hacemos la enseñanza. En otras palabras, es desde el actuar del maestro en su contexto de aula como podemos incidir de manera más significativa en el desarrollo del pensamiento crítico en los estudiantes.

Son múltiples los estudios que en la actualidad se han realizado en cuanto al pensamiento crítico en muy diferentes niveles de la educación. Algunos realizan caracterizaciones más del lado de la filosofía o de la psicología y concluyen, los primeros, con conceptualizaciones referidas a los procesos y productos característicos de las diferentes formas de construcción del conocimiento, mientras los segundos se refieren de manera más particular a describir y comprender los principales procesos de pensamiento de los sujetos. Así mismo, los diferentes estudios muestran una variedad importante en cuanto a los acercamientos metodológicos desde los cuales han realizado sus hallazgos y sobre los distintos contextos en los que se ha estudiado el pensamiento crítico, como quedará claro a lo largo del texto.

En el ámbito de las ciencias naturales, se suelen considerar como sinónimos el pen- samiento crítico y pensamiento científico ${ }^{1}$. Las investigaciones realizadas alrededor del pensamiento científico en niños (Spelke, 1991; Puche, 2000) demuestran que desde que ellos nacen poseen destrezas cognitivas que los hacen sensibles a ciertas propiedades de los objetos, así como a ciertas reglas físicas que los rigen (Spelke, 1991), y que facilitan posteriormente su desempeño en contextos naturales o en los contextos educativos formales.

Trabajos de investigación recientes desde la enseñanza de las ciencias sugieren la necesidad de incorporar dimensiones diferentes de la conceptual en los procesos de enseñanza y de aprendizaje (Caravita y Hallden, 1994; Tamayo, 2001, 2009). De igual manera, se considera necesario incorporar dimensiones como la afectiva y la creativa (Lipman, 1998), así como la realización de acciones que promuevan la perplejidad y el asombro en los niños. En esta línea de pensamiento, son abundantes las propuestas eduativas orientadas a formar a los profesores en procesos que faciliten el desarrollo de habilidades de pensamiento de orden superior en sus estudiantes, tales como: el análisis, la inferencia, la resolución de problemas, la toma de decisiones, la argumentación y la autorregulación, entre otras (Facione, 2007).

Laskey y Gibson (1987), citados por Guzmán y Sánchez (2006), plantean que el pensamiento crítico hace referencia a una compleja serie de actividades cognitivas que actúan conjuntamente, tales como la re-

\footnotetext{
No se pretende en este texto realizar una discusión detallada acerca de las semejanzas o diferencias entre el pensamiento crítico y el científico. Nos interesa en este momento reconocer que en la literatura especializada se pueden encontrar discusiones importantes sobre estos dos conceptos, las cuales desbordan el interés de este artículo. A lo largo del texto usaremos de manera preferente el término pensamiento crítico.
} 
solución de problemas, pensamiento lógico, percepción de ideas, análisis, evaluación y toma de decisiones. Los autores plantean la posibilidad de desarrollar el pensamiento crítico a través del planteamiento de diferentes tipos de preguntas en el aula, tales como aquellas destinadas a: recordar, interpretar, aplicar, analizar, sintetizar y evaluar. Para Ennis (1985), el pensamiento crítico es un pensamiento acertado y reflexivo, orientado en decidir qué pensar y qué hacer. Requiere llevar a cabo acciones como: juzgar la credibilidad de las fuentes, identificar las conclusiones, razones y supuestos, juzgar la calidad de un argumento incluyendo la aceptabilidad de sus razones, supuestos y evidencias, desarrollar una posición independiente acerca de un asunto, hacer preguntas clarificadoras adecuadas, planificar y diseñar experimentos, definir términos de manera apropiada para el contexto, tener apertura mental, tratar de estar bien informado y sacar conclusiones de forma cuidadosa y cuando se tenga la evidencia para hacerlo.

Movilizarnos de una enseñanza centrada en la transmisión de conceptos a otra que busca el desarrollo del pensamiento crítico en los estudiantes exige, entre otros aspectos, los siguientes:

- Reconocer en la construcción del sujeto su historia, su experiencia, sus intereses, su cultura. En términos de Facione (2007), el pensamiento crítico apareció mucho antes de que se inventara la escolaridad, yace en las raíces mismas de la civilización.

- Comprender las relaciones entre la ciencia y su conocimiento público (Fensham y Harlem, 1999; de Vos y Reiding, 1999;
Cross, 1999) y las relaciones entre ciencia, tecnología, sociedad y desarrollo.

- Generar procesos conscientes en el aprendizaje de las ciencias, como mecanismos que permitan profundizar y comprender cómo aprende el sujeto, para articularlo a procesos de enseñanza más significativos y en los que los procesos de autorregulación sean centrales (Tamayo, 2006).

- Considerar la escuela como un escenario que brinda la posibilidad no solo de acceder al conocimiento, sino también como el espacio en donde el niño enriquece su intelecto y donde recoge aportes fundamentales para construir o reconstruir el conocimiento de manera consciente.

- Orientar acciones hacia la construcción de ciudadanos autónomos.

Para Bachelard (1994), en la formación del espíritu crítico (científico) intervienen diferentes aspectos, dentro de los que se pueden citar: el sentido común, la intuición, el uso de imágenes, analogías, metáforas, el uso de generalizaciones, etc. Estos aspectos los plantea el autor como obstáculos que deben ser superados en la formación del espíritu científico; en este sentido, Mockus (1989) propone que privilegiar en el ámbito educativo la discusión racional, la tradición escrita y la reorganización de la acción se ha convertido en la actualidad en una potente herramienta que permite el desarrollo del pensamiento crítico, dinamizador por excelencia del conocimiento y de la formación de los sujetos.

Con las ideas presentadas hasta el momento, quedan enunciadas muchas de las 
múltiples perspectivas que hasta hoy se han tenido en cuenta para conceptualizar el pensamiento crítico. En las páginas siguientes nos centraremos en el estudio de una de las dimensiones constituyentes del pensamiento crítico: la argumentación².

\section{LA ARGUMENTACIÓN EN LA FORMACIÓN DEL PENSAMIENTO CRÍTICO}

Uno de los componentes del pensamiento crítico que se reconoce hoy como determinante incorpora la dimensión del lenguaje $\mathrm{y}$, de manera particular, la argumentación. El estudio del lenguaje y la argumentación en ciencias se constituye en la actualidad en una de las líneas de investigación de mayor prioridad en la didáctica de las ciencias (Lemke, 1997; Sutton, 1998; Candela, 1999). En cuanto a la argumentación en las clases de ciencias, Duschl y Osborne (2002) destacan la importancia de desarrollar investigaciones que permitan que los estudiantes se acerquen desde sus aulas de clase a las formas de trabajo científico propias de las comunidades académicas, dentro de las que se destaca, de manera especial, las referidas a los múltiples usos del lenguaje y de la argumentación. De otra parte, Jiménez y Díaz de Bustamante (2003), Campaner y De Longhi (2007), Sardá, Márquez y Sanmartí (2005), destacan el ámbito de la enseñanza de las ciencias como un espacio en el cual se pueden potenciar las competencias ar-

2 Proponemos el estudio del pensamiento crítico a traves de cuatro dimensiones: argumentación, metacognición, solución de problemas y emociones. Así mismo, consideramos el pensamiento critico como una habilidad de dominio específico que puede ser desarrollada en la escuela en el interior de cada uno de los diferentes campos disciplinares estudiados o mediante el estudio inter y transdisciplinar de los diferentes problemas empleados en la enseñanza. Las interacciones entre las cuatro dimensiones aquí mencionadas adquirirían sentidos difererntes, en cuanto a la acción de pensar críticamente, en el interior de un campo específico del conocimiento (Tamayo et ál., en prensa). gumentativas de los estudiantes, dado que uno de los fines de la investigación científica es la generación y justificación de enunciados y acciones encaminados a la comprensión de la naturaleza (Jiménez, Bugallo y Duschl, 2000 (citado en Jiménez y Díaz de Bustamante, 2003).

Los estudios sobre la argumentación en el aula muestran en general dos tendencias: los de orden estructural, que profundizan en la comprensión de las formas de los argumentos; y los de orden funcional, que tienen como intención entender los usos de la argumentación. En la enseñanza de las ciencias, varios autores han analizado la argumentación en el contexto del aula basándose en los modelos propuestos por Toulmin, Van Dijk y Adam. El primero tiene su origen en teorías de razonamiento práctico y se refiere a la práctica jurídica y los segundos a la lingüística textual (Sardá y Sanmartí, 2000). Toulmin considera como argumento todo aquello que es utilizado para justificar o refutar una proposición. Aunque no ofrece los rasgos lingüísticos de su modelo, estos han sido inferidos a partir de los elementos funcionales de aquel (Parodi, 2005). El modelo de Toulmin permite que los alumnos reflexionen sobre la estructura del texto argumentativo (Sardá y Sanmartí, 2000; Driver y Newton, 1997).

Van Dijk (1989) sostiene que la estructura del texto argumentativo puede ser descompuesta más allá de la hipótesis (premisas) y la conclusión, e incluye la justificación, las especificaciones de tiempo y lugar y las circunstancias en las cuales se produce la argumentación. Para él, lo que define un texto argumentativo es la finalidad que este tiene de convencer. El autor caracteriza en un texto argumentativo tres niveles de or- 
ganización: la superestructura, la macroestructura y la microestructura. El estudio de los diferentes niveles de la estructura del texto argumentativo puede favorecer, en las clases de ciencias, la apropiación de las características del lenguaje científico. Adam (1995) muestra, por su parte, cómo un texto argumentativo puede estar estructurado en diferentes secuencias. Tomando como base el modelo de Toulmin, Adam analiza los textos argumentativos como secuencias argumentativas encadenadas en las que se puede dar el caso de que la conclusión de una secuencia sea la premisa de la siguiente. De otra parte, para Revel, Couló, Erduran, Furman, Iglesia y Adúriz-Bravo (2005), la argumentación es una actividad social, intelectual y verbal que sirve para justificar o refutar una opinión, y que consiste en hacer declaraciones teniendo en cuenta al receptor y la finalidad con la cual se emiten. Para Perelman y Olbrechts-Tyteca (1989), la argumentación está orientada hacia el convencimiento o la persuasión, en tanto consideran que la finalidad de la argumentación es convencer con razones o persuadir mediante recursos afectivos.

A partir de estos modelos, y mediante la elaboración de un esquema que articula varios aspectos de algunos de ellos, Sardá y Sanmartí (2000) estudiaron la argumentación en estudiantes de ciencias que participaron en un juego de rol. Las autoras encontraron que los patrones estructurales de los argumentos eran completos y que había uso adecuado de los conectores lógicos. Sin embargo, el análisis funcional del texto mostró dificultades relacionadas con la relevancia y pertinencia de los argumentos, la elección de evidencias desde teorías implícitas más que científicas, interpretaciones e inferencias no justificadas y conclusiones no derivadas del contexto teórico. En el aula de clase este proceso se evidenciaría a través de las prácticas discursivas de los estudiantes en las que se articulan componentes de la estructura de la argumentación, de los conceptos científicos y de la práctica discursiva, cuya puesta en escena permitiría conocer las características de los modelos argumentativos y, a partir de allí, construir procesos didácticos que contribuyan a la transformación de dichos modelos.

De otra parte, la argumentación, como lo expresan Driver y Newton (2000) cuando definen la argumentación dialógica o de voz múltiple, tiene lugar cuando se examinan, por un individuo o en el seno de un grupo, diferentes perspectivas para llegar a un acuerdo sobre cuáles son las afirmaciones del conocimiento que se aceptan o las líneas de acción que se toman en consideración. En un sentido similar, Candela (1999) destaca la importancia de la argumentación en el aula en función de la construcción de la búsqueda de acuerdos $\mathrm{y}$, en última instancia, de consensos, aspectos que pueden ser complementarios y parten de una misma intención. Frecuentemente se argumenta para convencer de la validez de una versión del conocimiento $\mathrm{y}$, por tanto, para llegar después a consensos. En relación con este último aspecto, Giere (1992) plantea que la argumentación en ciencias es un proceso de elección entre modelos y teorías para explicar los fenómenos de la realidad, proceso al cual se puede llegar si se generan interpretaciones diferentes de los datos debido a las interpretaciones particulares de las comunidades científicas, a los avances tecnológicos y a los cambios en los objetivos de las ciencias (Duschl y Gitomer, 1997). 
Considerar la argumentación como constituyente del pensamiento crítico nos lleva a orientar acciones en función de comprender cómo profesores y estudiantes argumentan en el aula. En tal sentido, nos proponemos, en lo que sigue de este documento, identificar las principales características de los procesos argumentativos como constituyentes del pensamiento crítico en niños de educación básica primaria y diseñar.

\section{Metodología}

La investigación sigue un enfoque descriptivo-comprensivo que tuvo como propósito caracterizar los procesos y productos argumentativos en niños de educación básica primaria. Se diseñaron y aplicaron 10 actividades de enseñanza, las cuales fueron resueltas por los estudiantes. A partir de ellas se realizaron análisis cuantitativos y cualitativos, los cuales permitieron describir comprensivamente los procesos argumentativos realizados por los estudiantes. Participaron en la investigación 2.200 niños y niñas de los grados $4^{\circ}$ y $5^{\circ}$ de básica primaria. Los análisis que presentamos a continuación fueron realizados con 220 niños seleccionados aleatoriamente. El análisis se realizó en tres momentos: en el primero se trianguló información con los instrumentos 1, 2 y 3; en el segundo, con los instrumentos 4, 5 y 6, y en el tercero con los instrumentos 7, 8 y 9. El instrumento 10, dedicado de manera específica a solución de problemas, no fue tenido en cuenta en los resultados aquí presentados.

Para el análisis de la información se tomaron como punto de partida algunos de los aportes derivados de las matrices argumentativas presentadas por Toulmin (2007), con el fin de crear las categorías que serán el punto de partida en el presente informe de investigación. De acuerdo con Erdurán et ál. (2004) y Erduran (2008), la calidad de los argumentos se puede evaluar desde los siguientes niveles argumentativos (tabla 1).

Tabla 1. Niveles argumentativos

\begin{tabular}{|l|l|}
\hline \multicolumn{1}{|c|}{$\begin{array}{c}\text { Niveles } \\
\text { argumentativos }\end{array}$} & \multicolumn{1}{c|}{ Características } \\
\hline Nivel 1 & $\begin{array}{l}\text { Comprende los argumentos que son una descripción simple de la } \\
\text { vivencia. }\end{array}$ \\
\hline Nivel 2 & $\begin{array}{l}\text { Comprende argumentos en los que se identifican con claridad los datos } \\
\text { (data) y una conclusión (claim). }\end{array}$ \\
\hline Nivel 3 & $\begin{array}{l}\text { Comprenden argumentos en los cuales se identifican con claridad los } \\
\text { datos (data), conclusiones (claim) y justificación. }\end{array}$ \\
\hline Nivel 4 & $\begin{array}{l}\text { Comprende argumentos constituidos por datos, conclusiones y justifica- } \\
\text { ciones (warrants), haciendo uso de cualificadores (qualifiers) o respaldo } \\
\text { teórico (backing). }\end{array}$ \\
\hline Nivel 5 & $\begin{array}{l}\text { Comprende argumentos en los que se identifican datos, conclusión(es), } \\
\text { justificación(es), respaldo(s) y contraargumento(s). }\end{array}$ \\
\hline
\end{tabular}




\section{ANÁlisis, Discusión Y CONCLUSIONES}

En la tabla 2 puede observarse que el 19,9\% del total de respuestas analizadas corresponde al nivel 1, el 46,8\% al nivel 2, el 31,3\% al nivel 3, el 3,7\% al nivel 4 y el 1,4\% al nivel 5. En cuanto a los momentos de la in- vestigación, los niveles argumentativos con mayores frecuencias en las respuestas, en el momento 2 , son los niveles 2 y 3 con $23,5 \%$ y $68,6 \%$, respectivamente. En el momento 3 se destaca la alta frecuencia de respuestas $(73,1 \%)$ correspondientes al nivel argumentativo 3 .

Tabla 2. Distribución de frecuencias de los diferentes niveles argumentativos encontrados

\begin{tabular}{|l|c|c|c|}
\hline & Momento 1 & Momento 2 & Momento 3 \\
\hline Nivel 1 & 19,9 & 3,7 & 4,2 \\
\hline Nivel 2 & 46,8 & 23,5 & 13,2 \\
\hline Nivel 3 & 31,3 & 68,6 & 73,1 \\
\hline Nivel 4 & 3,7 & 4,1 & 9 \\
\hline Nivel 5 & 1,4 & 0 & 0,6 \\
\hline
\end{tabular}

El análisis de las frecuencias en las respuestas de los estudiantes según los diferentes niveles argumentativos muestra cierta tendencia al empleo de niveles argumentativos más exigentes a medida que la intervención de aula avanza. Es decir, como se evidencia en la tabla 2, que mientras las frecuencias de los niveles 1 y 2 disminuyen en los tres momentos de la investigación, las frecuencias en el nivel argumentativo 3 se incrementan hasta el nivel 3. En otras palabras, los estudiantes dejan de emplear con tanta frecuencia los niveles argumentativos 1 y 2 . Este movimiento hacia niveles argumentativos de mayor exigencia para los estudiantes se deriva posiblemente del trabajo intencionado realizado por los profesores en función del desarrollo de ciertas habilidades argumentativas en los estudiantes, a partir del conjunto de actividades desarrolladas a lo largo de la intervención didáctica (ver anexo).
Pasar de estructuras argumentativas en las cuales los estudiantes realizan descripciones simples de experiencias (Ericsson y Kintsch, 1995) con el empleo de verbos que implican vivencias concretas (observé, toqué, sentí...), a estructuras argumentativas donde los estudiantes identifican con cierta claridad los datos y la conclusión contenidos en la situación presentada y, posteriormente, a otras en las que, ademas de identificar datos y conclusión, pueden incluir una o varias justificaciones, parece ser un logro importante en función de desarrollar habilidades o competencias argumentativas. Este tránsito de un modelo argumentativo a otro tiene como características importantes las siguientes:

1. Descentramiento de la experiencia directa e inmediata en la cual se encuentran los estudiantes, a otra dimensión en la cual adquiere importancia cierta actividad intelectual de ellos. Es decir, 
tránsito hacia la construcción de argumentos distanciados del ámbito de la experiencia concreta en la que se presenta la actividad sobre la cual se requiere la construcción argumentativa.

2. Cierto abandono de la descripción simple de la actividad presentada, lo cual puede estar acompañado de la diferenciación entre datos y conclusión. Es decir, el paso de estructuras argumentativas en las cuales los estudiantes no diferencian datos de conclusión y describen literalmente la actividad presentada, a estructuras argumentativas donde los estudiantes identifican con claridad los datos y las conclusiones $\mathrm{y}$, a su vez, proponen justificaciones para las situaciones presentadas.

3. La presencia de las justificaciones parece constituirse en un indicador de extrema importancia al valorar la calidad del argumento expresado por los estudiantes. Si entendemos la justificación como la búsqueda de las razones que en última instancia explican el fenómeno estudiado, incluir justificaciones en los argumentos supone adquirir el compromiso de explicitar el paso de los datos a la conclusión.

Con base en la tabla 2, podemos realizar los siguientes análisis complementarios. El $63,6 \%$ de los argumentos elaborados por los estudiantes en el momento 1 se ubicaron en los niveles argumentativos 1 y 2, mientras el porcentaje de los argumentos en estos mismos niveles en el momento 3 fue de $17,4 \%$. El 31,3\% de los argumentos elaborados por los estudiantes en el momento 1 se ubicaron en el nivel argumentativo 3, mientras el $73,1 \%$ de los argumentos elaborados por los estudiantes en el momento 3 se ubicaron en el nivel argumentativo 3. Estos dos hallazgos evidencian cierto tránsito de modelos argumentativos menos elaborados y centrados en la identificación de datos y conclusión a modelos más elaborados en los que, además de identificar datos y conclusión, se justifican las observaciones realizadas.

A continuación presentamos un análisis más detallado de los argumentos elaborados por los estudiantes para cada uno de los cinco niveles argumentativos encontrados.

El nivel 1 de argumentación comprende los argumentos que son una descripción simple de la vivencia (Ericsson y Kintsch, 1995). Utiliza en su ejercicio verbos como observé, toqué, froté, sentí, pero se limita a explicar lo que ocurrió en la realización de los experimentos. El estudiante se enfoca solo en describir los datos de lo que ocurrió en la actividad. Todas las respuestas que hacen parte del nivel 1 de argumentación se caracterizan por realizar descripciones literales de los fenómenos observados, que llevan al lector a recrear la escena de realización de los experimentos. Para tal efecto, el estudiante hace uso de algunos verbos en primera persona, con el fin de describir con detalle lo que se hizo en la actividad. Los porcentajes de argumentos de nivel 1 encontrados en los tres momentos de la investigación fueron, respectivamente, $19,8 \%$, $3,7 \%$ y $4,2 \%$. Si bien no desaparecen estos argumentos constituidos solamente por descripciones literales de los fenómenos presentados, llamamos la atención sobre la disminución sustancial que se da entre los momentos 1 y 2. Al parecer, los estudiantes aprenden con cierta facilidad la estructura básica argumentativa que les permite identificar datos y conclusión, dejando atrás la 
descripción literal de los fenómenos presentados, tal como se ilustra a continuación:

\section{3-4-22-1-2-Porque el agua se estaba chorriando (sic) y la vela se estaba apa- gando y se estaba calentando el agua y yo la toqué y estaba caliente.}

\section{3-4-22-3-2-Yo sentí que se me levantaba} el pelo y empezamos a pasarla por la ropa y después la bomba se calentó y después se lo pusimos entonces en el pelo de mi compañero.

8-4-7-5-1- hoy en el primer vaso ella se quedó en la mitad. En el segundo vaso que se hundió le echamos la cucharada de sal. En el tercer vaso la papa se quedó flotando.

Los textos anteriores muestran de manera clara las descripciones realizadas por los estudiantes con base en la observación de los fenómenos presentados. Son descripciones literales que muestran lo sucedido en el experimento, que no arriesgan posibles explicaciones o justificaciones, centradas en la dimensión experiencial y, en cierta forma producidas con la mediación activa de los órganos de los sentidos, en lo que podríamos llamar, con Rivière (1988), un argumento con carácter fenoménico. En otras palabras, un argumento construido a partir de la apariencia externa del fenómeno estudiado, un argumento que describe el fenómeno, que lo recrea en su forma, sin explicaciones o justificaciones que nos den a entender algo acerca de su comprensión.

El análisis de los argumentos desde los distintos niveles representacionales propuestos por Ericsson y Kintsch (1995) nos puede ser útil en este momento para comprender mejor este primer nivel argumentativo encontrado en los estudiantes. Los autores citados proponen los siguientes tres niveles:
- Representación lingüística superficial, la cual es elaborada a partir de las palabras presentes en el texto original.

- Representación de la base del texto, en la cual se especifican las diferentes relaciones semánticas entre las distintas partes del texto. Es una representación proposicional de las ideas y los conceptos, donde las paráfrasis permiten al alumno conectar ideas de procedencia distinta de la del texto original y establecer niveles jerárquicos entre ellas.

- Representación del modelo situacional, equivalente a la coherencia global del texto, en la cual se ubican las referencias y las oraciones nuevas. Es una representación de la situación real que el texto describe $\mathrm{y}$, por tanto, puede soportar reelaboraciones muy alejadas del texto original; son el resultado de la comprensión del contenido principal del texto.

Los textos ubicados en el nivel argumentativo 1 representan, en el mejor de los casos, la situación dada en los diferentes experimentos y actividades de aula. Su característica central es el empleo de las mismas expresiones utilizadas en la situación presentada, de tal manera que los estudiantes terminan parafraseando los textos leídos y escuchados. En este caso no observamos que los estudiantes demuestren comprensión de la situación, lo cual se corrobora con el empleo de lenguajes descriptivos en los que se narra la actividad dada. Más que lograr comprensiones cabales del las diferentes situaciones presentadas, los estudiantes describen las actividades fenoménicamente a partir de acciones senso-perceptuales, lo cual se concreta con el empleo de verbos en primera persona que hacen referencia a lo 
sentido por ellos en el transcurso de la actividad. El predominio de acciones mediadas por los sentidos (oír, tocar, sentir...) se complementa con la ausencia de acciones que evidencien esfuerzos de parte de los estudiantes en función de comprender las distintas situaciones dadas. La presencia de lo fenoménico unido a la ausencia de lo reflexivo en el actuar de los estudiantes los lleva, en términos de producción textual, a describir de manera tautológica lo observado y, en consecuencia, a evidenciar comprensiones superficiales o descripciones literales de las diferentes actividades diseñadas para explorar sus habilidades y competencias argumentativas.

El nivel 2 de argumentación comprende argumentos en los que se identifican con claridad los datos (data) y una conclusión (claim):

31-4-5-6-1-2-Porque es como una hoja delicada y se quemará todo lo que es papel.

6.4 ¿Por qué en los tres péndulos las bolas se mueven a distinta velocidad? (D), a lo que contesta: "porque uno hizo más fuerte" (C), concluyendo, al parecer, que el comportamiento de los péndulos era mediado por la fuerza que le imprimía el estudiante al realizar el ejercicio.

En este nivel argumentativo se destaca el empleo de, al menos, una conclusión en los argumentos de los estudiantes. Frente al nivel argumentativo anterior, la diferencia reside en la presencia o no de conclusiones. En este caso, los estudiantes no describen literalmente el fenómeno y no solo enumeran o identifican los datos contenidos en las actividades presentadas; por el contrario, empiezan a identificar posibles conclusiones derivadas de los datos identificados. Al parecer, distinguir las descripciones literales de los datos de las conclusiones se logra con cierta facilidad, lo cual se evidencia en los resultados encontrados entre el primer y el segundo momento de la investigación (tabla 2). Identificar datos y conclusión se constituye, entonces, en la estructura argumentativa más simple, la cual empieza a evidenciarse en este nivel. Derivar conclusiones de una serie de datos exige, al menos, las siguientes acciones de parte de los estudiantes:

- Identificar los datos.

- Identificar las conclusiones.

- Establecer diferencias entre un dato y una conclusión.

- Establecer ciertas relaciones, causales o no, entre datos y conclusión.

- Dar cierto orden, cierta estructura, al proceso de pensamiento empleado.

- Regular, de manera consciente o no, la relación entre datos y conclusión.

- Adquisición de cierto compromiso en función de mirar la situación de manera integrada.

- Asumir cierto reto cognitivo-lingüístico en el cual, de una parte, debe elaborar un producto textual, un argumento $\mathrm{y}$, de otra, relacionar de alguna manera datos en función de una conclusión. Es decir, en este caso el estudiante reconoce cierta demanda cognitiva y conceptual de la situación presentada, distinta de la demanda sentida frente a la descripción de la situación, o de aquella centrada en la identificación de los datos, presente en 
el nivel argumentativo 1. Queda obviamente por establecer la conciencia que tienen los estudiantes sobre este reto asumido, la conciencia de la demanda cognitiva de la tarea y de la producción del argumento con la participación de datos y conclusión.

De las acciones antes descritas, unas estructurales y otras funcionales, derivamos el nivel argumentativo de los estudiantes. Forma y contenido, estructura y función, se hacen una en el argumento y son una, así mismo, en el proceso de argumentación. No obstante lo anterior, nos interesa asumir una perspectiva funcional en el estudio de los argumentos elaborados por los estudiantes, obviamente sin desconocer la misma estructura argumentativa.

El nivel 3 de argumentación comprende argumentos en los cuales se identifican con claridad los datos, conclusiones y justificación. En las intervenciones que sustentan este nivel argumentativo se destacan las que poseen datos, varias conclusiones de los experimentos desarrollados y una o varias justificaciones de sus argumentos, expresando de manera fluida y coherente sus ideas. Además de lo anterior, son argumentos mejor estructurados, donde se visualizan conectores, buen manejo de vocabulario, redacción clara y de fácil interpretación, como se ilustra en los siguientes textos:

12-5-7-4-2-la bola quedó flotando y al momento se cayó [...] porque uno flota y el otro se va hasta abajo y se hunde.

32-5-34-7-3-No es posible porque está muy oscuro y el arcoíris se ve cuando llueve.

12-5-7-6-4- porque una tiene una cuerda más pequeña la otra la tiene grande.
El aspecto que establece la diferencia con el nivel argumentativo anterior es la presencia de justificaciones. Mientras en el nivel 2 los estudiantes identifican datos y conclusión, en el 3 identifican datos, conclusión y dan las explicaciónes (justificaciones) a los fenómenos en cuestión. Además de esta diferencia, encontramos que los textos realizados por los estudiantes son más extensos y cualitativamente mejor elaborados. Centraremos nuestro análisis en la importancia de la presencia de las justificaciones en los modelos argumentativos de los estudiantes. Frente a este aspecto resultan interrogantes como: ¿cuál es la función de las justificaciones en los argumentos realizados por los niños?, ¿por qué la justificación de las situaciones presentadas nos permite suponer un cambio cualitativo determinante en la construcción de los argumentos?, ¿cuáles son las posibles relaciones entre un modelo argumentativo con datos, conclusión y justificación en la comprensión de los fenómenos estudiados?, ¿qué tipos de justificaciones realizan los estudiantes? A continuación nos referiremos a algunas de estas preguntas.

Zohar y Nemet (2002) evalúan la calidad de los argumentos escritos por los estudiantes según su estructura y contenido. Un argumento fuerte tiene varias justificaciones que soportan una conclusión, la cual incorpora conceptos científicos y hechos adecuados, relevantes y específicos. Los argumentos débiles consisten en justificaciones individuales no relevantes. Para los autores, las conclusiones que no incluyen algún tipo de justificación no son consideradas como argumentos. Los criterios que proponen para la clasificación de las justificaciones son: a) no consideración de conocimiento científi$\mathrm{co}, \mathrm{b})$ inadecuado conocimiento científico, 
c) conocimiento científico no específico y d) conocimiento científico correcto.

En función de identificar un marco para juzgar la calidad de los argumentos elaborados por los estudiantes, Sandoval y Millwood (2005) proponen criterios basados en la dependencia de campo. De manera específica, se refieren a dos dimensiones de la argumentación científica: en la primera evalúan la calidad conceptual, la cual incluye la pertinencia de las conclusiones causales dentro de un marco teórico específico y la justificación de esas conclusiones con el uso de los datos disponibles. En la segunda evalúan la calidad epistemológica, la cual está constituida por tres componentes: a) citar suficientes datos que justifiquen la conclusión, b) escribir una explicación causal coherente para el fenómeno dado y c) incorporar apropiadas referencias retóricas cuando nos referimos a los datos.

Un aspecto importante en los planteamientos de Sandoval y Millwood es que evalúan la posibilidad de generar argumentos que expliquen ciertos fenómenos en el marco de una teoría específica, lo cual de alguna manera incorpora los criterios propuestos por Zohar y Nemet, descritos anteriormente, para clasificar las justificaciones. Además de lo anterior, el marco teórico que proponen da información acerca de los criterios epistemológicos usados por los estudiantes cuando generan argumentos y de cómo estos criterios están alineados con los criterios empleados dentro de un dominio científico específico. Los autores sugieren que argumentos de alta calidad requieren comprensión conceptual de las relaciones entre las teorías científicas y su relación con su aplicación a la solución de problemas específicos, así como la comprensión epistémica de los criterios que determinan argumentos de alta calidad. La manera como los estudiantes incorporan los datos en sus argumentos y escriben las explicaciones de los fenómenos muestra sus creencias epistemológicas frente a los fenómenos estudiados.

La identificación de los datos, la conclusión y la justificación frente a un fenómeno determinado no es garantía de argumentos fuertes o de alta calidad. Aunque los estudiantes comprendan la importancia de relacionar datos y conclusión, es frecuente que ellos tiendan a enfatizar sobre un conjunto limitado de datos en función de apoyar una conclusión determinada. Los estudiantes usualmente no incluyen la comparación de datos de diferentes fuentes cuando justifican una conclusión, siendo esta comparación necesaria, lo cual llevó a Mani-Ikan a considerar la importancia de encontrar puntos de vista contradictorios $\mathrm{u}$ opuestos en el proceso de evaluar la calidad de los argumentos (Mani-Ikan, 2005, citado en Schwarz, 2009).

También es frecuente encontrar que los estudiantes realicen explicaciones coherentes en el marco de ciertos principios teóricos; es decir, que consideren que sus explicaciones son válidas a la luz de una teoría determinada (Sandoval, 2001), lo cual puede comprenderse a partir de las características de sus ideas previas y de sus modelos, desde los cuales encuentran coherencia entre lo observado y la explicación construida o, en términos más rigurosos, observan la realidad a partir de ciertos modelos que han construido ontológicamente, lo cual los lleva a encontrar la coincidencia antes señalada.

Los argumentos de nivel 3 encontrados en los estudiantes investigados están consti- 
tuidos por datos, conclusión y justificación. Sin embargo, es claro que las justificaciones elaboradas distan sustancialmente de lo propuesto por Zohar y Nemet (2002), Sandoval y Millwood (2005) y Mani-Ikan (2005, citado en Schwarz, 2009). Son argumentos en los cuales no encontramos justificaciones sólidas desde campos conceptuales específicos, ni confrontaciones entre diferentes puntos de vista en el proceso de construir el argumento. Lo anterior seguramente se debe al nivel educativo en el cual se realizó la investigación (grados 4 y 5 de educación básica primaria). No obstante lo anterior, encontramos en este nivel argumentativo que los estudiantes reconocen que el argumento debe contener los tres componentes antes mencionados: datos, conclusión y justificación. Encontrada esta estructura básica del argumento en los estudiantes, nos queda por enfatizar en la necesidad de enriquecerla funcionalmente, lo cual requiere acciones en diferentes perspectivas en función de lograr que los estudiantes elaboren argumentos fuertes de las situaciones que se les presentan. A continuación enunciamos algunos aspectos que, sin lugar a dudas, pueden orientar a los profesores en función del desarrollo de habilidades argumentativas en los estudiantes.

- Orientar acciones de enseñanza dirigidas a diferenciar claramente datos, conclusiones y justificaciones.

- Desarrollar habilidades metacognitivas en los estudiantes en función del logro de procesos concientes e intencionados con el propósito de distinguir datos de conclusiones y justificaciones.

- Aplicar esta estructura argumentativa básica al estudio de diferentes fenóme- nos y situaciones propias del ámbito de las ciencias.

- Adquirir gradualmente la terminología propia del campo.

- Desarrollar habilidades de pensamiento lógico útiles para el aprendizaje de las ciencias.

- Desplazar la atención del fenómeno concreto estudiado hacia las relaciones causales abstractas que lo explican.

- Comprender algunas de las relaciones existentes entre pensamiento científico y pensamiento de sentido común.

- Adquirir habilidades para explicar científicamente fenómenos cotidianos.

El nivel 4 de argumentación comprende argumentos constituidos por datos, conclusiones y justificaciones, con el empleo de cualificadores o respaldo teórico. En este nivel argumentativo son de especial importancia los respaldos teóricos en los textos escritos por los estudiantes. De acuerdo con la propuesta de Toulmin, un esquema para analizar los argumentos relaciona los datos con la conclusión, para lo cual se requiere de las garantías y estas, a su vez, se apoyan en otras certezas, sin las cuales las propias garantías carecerían de autoridad (Toulmin, 2007). La presencia de la garantía en el argumento nos lleva a preguntarnos acerca de su pertinencia, de su aplicabilidad a un caso particular y de su posible aceptación general. En otras palabras, la presencia en la estructura argumentativa de datos, conclusión y garantía no nos asegura un argumento coherente. Así mismo, la presencia de respaldos de nuestras garantías y su uso adecuado en el marco de un campo del sa- 
ber específico se constituye en un punto crítico para la evaluación de los argumentos. Los enunciados de las garantías son hipotéticos, mientras los respaldos de las garantías pueden expresarse como enunciados categóricos sobre hechos.

Figura 1. Estructura argumentativa general propuesta pot Toulmin

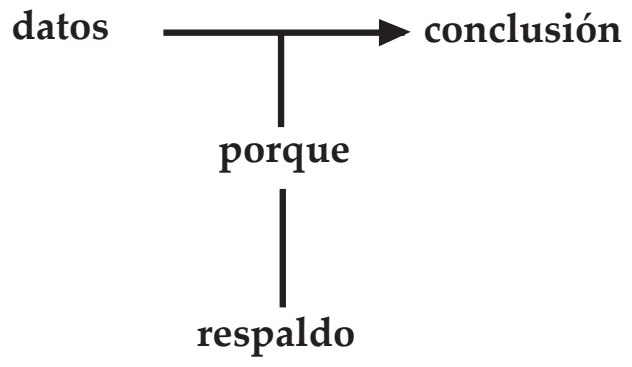

Ya en páginas anteriores nos hemos referido a datos y conclusión como constituyentes de los argumentos elaborados por los niños participantes en la investigación. A continuación dedicaremos algunas líneas a las garantías y a los respaldos presentes en los textos por ellos elaborados. Para Toulmin, el tipo de respaldo que apoya una garantía varía de un campo disciplinar a otro, de tal manera que los respaldos empleados para soportar las garantías en ciencias naturales son diferentes a los respaldos empleados en ciencias sociales. Plantea el autor la variabilidad o dependencia del campo, del respaldo o fundamento necesario para establecer la garantía (Toulmin, 2007: 141). Con las precisiones conceptuales realizadas hasta el momento pasamos a continuación a referirnos al análisis de las garantías y respaldos elaborados por los niños frente a las diferentes situaciones presentadas, no sin antes aclarar que discusiones en torno a los matizadores modales y condiciones de excepción, de las que habla el autor, no serán tenidas en cuenta aquí debido a que no fueron encontradas en los datos recogidos.

Los textos que presentamos a continuación ilustran el nivel argumentativo 4, conformado por argumentos completos, bien estructurados en su forma y finalidad, con ideas claras, buen uso del vocabulario, uso de cualificadores o modalizadores, o bien con la presencia de un respaldo teórico que fortalece su desempeño en la actividad argumentativa.

55-4-3-6-1- la más pequeña realizó más recorridos y la más grande menos recorridos, y yo creo que fue por el ciclo entre más grande y más pequeña.

55-4-3-6-2-porque unas bolas tienen el hilo más largo y entonces creo que el hilo entre más pequeño más rápido se mueve la bola.

49-5-1-7-5-Del agua y del sol, entonces cuando se juntan los rayos del sol y las goteras de agua van los colores del arcoíris.

Los textos presentados contienen datos, conclusión, justificación y respaldo teórico; tal es el caso de 55-4-3-6-2, cuando afirma que el movimiento más rápido de las bolas en el experimento del péndulo se debe a la longitud del hilo, hasta llegar a plantear que entre más pequeño (corto) el hilo, más rápido se mueve la bola (masa del péndulo). En las respuestas 55-4-3-6-1 y 49-5-1-7-5 vemos que los estudiantes tratan de respaldar sus justificaciones en principios teóricos referidos a los ciclos del movimiento pendular y a la refracción de la luz a su paso por las gotas de agua; sin embargo, es claro que estos argumentos son aún muy incipientes.

Si bien en los textos presentados hallamos un modelo argumentativo constituido por 
datos, conclusión, justificación y respaldo, es claro que, en su conjunto, el argumento es débil. El respaldo conceptual que soporta la justificación no es preciso en ninguno de los casos mencionados. Cuando el estudiante, al referirse al péndulo, plantea "[...] que el hilo entre más pequeño más rápido se mueve la bola", reconoce la relación causal entre la longitud del péndulo y la frecuencia; sin embargo, no explora desarrollos conceptuales que no estén mediados por lo observado en el experimento. No se dan respaldos teóricos en relación, por ejemplo, con el periodo y con la amplitud. En el caso de la actividad referida al arcoíris el estudiante plantea que los colores surgen cuando los rayos de sol se juntan con las gotas de agua, sin llegar a identificar las condiciones necesarias para la formación del arcoíris. Podríamos decir, en este caso, que los estudiantes no identifican las variables responsables del fenómeno observado.

El nivel 5 de argumentación muestra argumentos en los que se identifican datos, conclusión(es), justificación(es), respaldo(s) y contraargumento(s). Las respuestas dadas por los estudiantes y que se ubican en este nivel argumentativo se caracterizan por presentar, además de lo descrito en los otros niveles, contraargumentos. Solo el 0,66\% de los niños presentaron argumentos en este nivel.

5-4-6-1-2-porque el papel al hacer contacto se quema con la llama de la vela.

5-4-6-2-3-pedí un carro para transportar, le hice una caja con rotos para que no se ahogara para poder llevarlo a mi casa sano y salvo.

5-4-6-2-4- le hice una caja con rotos, pero no debí hacerlo porque los animales necesitan la naturaleza, el aire, el sol, la lluvia y los amigos.
5-4-6-2-7- mis compañeros lo colocaron en un frasco, pero todos hicimos mal, debimos dejarlo en la naturaleza porque es el mejor hábitat donde ellos pueden estar.

5-4-6-3-2- porque la bomba hace contacto con el pelo.

En otros casos los estudiantes emplean moduladores, como ilustramos a continuación:

5-4-6-7-1-el de abajo sí porque está abajo, y el de arriba sí pero poquito.

5-4-6-7-3-no, no puede verse así llueva mucho.

5-4-6-7-4-el de abajo porque está abajo, el de arriba un poquito pero no creo porque está nublado.

5-4-6-7-5-cuando llueve mucho se nubla y sale el sol y se forma el arcoíris.

Al parecer son dos aspectos los que hacen difícil el tránsito del nivel argumentativo 4 al 5. En primer lugar, el empleo de moduladores $y$, en segundo lugar, la presencia de contraargumentos (figura 2). Ya en páginas anteriores mencionamos la importancia de presentar situaciones polémicas para favorecer la elaboración de contraargumentos (Mani-Ikan, 2005, citado en Schwarz, 2009).

Figura 2. Estructura argumentativa con el empleo de moduladores y contraargumentos

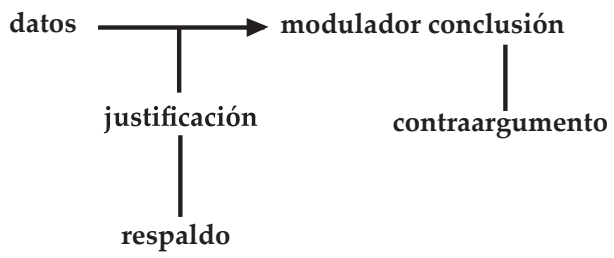

Las explicaciones, los respaldos teóricos $\mathrm{y}$, en última instancia, los argumentos, se constituyen en especies de cajas negras em- 
pleadas por los estudiantes para referirse a las situaciones presentadas, insinuando comprensiones superficiales de los fenómenos. En este sentido, la aprehensión de la situación a la que alude el problema presentado a los estudiantes está mediada por la apariencia externa del fenómeno. Los estudiantes identifican con cierta facilidad los datos proporcionados en la situación, se refieren a la conclusión, logran presentar justificaciones derivadas de la experiencia directa, pero no respaldan teóricamente estas justificaciones. La presencia de respaldos teóricos sólidos sin lugar a dudas es un aspecto que se logra con la escolarización, $\mathrm{su}$ ausencia es la principal responsable de argumentos débiles; en tal sentido, en la enseñanza se deben orientar esfuerzos de manera intencionada para conseguir que los estudiantes no solo aprehendan el fenómeno en su forma, sino, además, y lo que es más importante, que le permitan referirse a él en el plano abstracto.

Este movimiento de lo concreto a lo abstracto, independiente de la edad, permite que los estudiantes elaboren argumentos más sólidos, más fuertes, en el marco de los requerimientos propios del sistema educativo y del nivel de formación, lo cual requiere, de parte de los profesores, diseñar ambientes de enseñanza apropiados que permitan jalonar el desarrollo de los estudiantes.

A continuación nos referiremos, a manera de conclusiones, a algunos de los aspectos que podrían ser de gran interés para lograr procesos argumentativos más profundos y consistentes en los estudiantes.

- Diseño de ambientes de enseñanza y aprendizaje orientados al desarrollo de habiliades argumentativas en los estudiantes. En el marco de los desarrollos teóricos actuales (Toulmin, 2007; Jiménez-Alexandre, 2008; Erduran, 2008; Mercer, 2009; Schwarz, 2009; Muller, Perret-Clermont, Tartas y Iannaccone, 2009; Regh, 2010) se requiere que el actuar del maestro en las aulas de clase esté orientado a que el estudiante identifique y diferencie con claridad los datos de las conclusiones, las justificaciones, los respaldos teóricos y los contraargumentos. De igual manera, es necesario que tanto profesores como estudiantes comprendan que la argumentación en ciencias es condición sine qua non para el logro de comprensiones profundas de lo estudiado y, así mismo, es la actividad central del trabajo científico.

- En la práctica cotidiana de la argumentación en el aula intervienen diferentes dimensiones, las cuales interactúan de manera interdependiente. La argumentación involucra procesos cognitivos, interactivos y dialógicos, en torno a temas específicos y en el marco de contextos institucionales y culturales determinados. Algunas de las dimensiones que se deben tener en cuenta son: el individuo con sus propias capacidades cognitivas y comunicativas, los interlocutores con su estatus e intenciones, el tópico discutido, las herramientas usadas y el contexto sociocultural (Muller et ál., 2009). En cuanto a la dimensión intrapersonal de la argumentación nos referimos a las herramientas del pensamiento, a nivel individual, requeridas para participar en los procesos argumentativos; esto implica comprender los prerrequisitos cognitivos que les permiten a los sujetos participar en los procesos argumentativos, lo cual incluye la dimensión afectiva y las maneras como se relacionan 
los sujetos con los temas de discusión y con las herramientas de mediación en la construcción de los argumentos. En cuanto a la dimensión dialógica e interpersonal, se debe reconocer que la argumentación es una actividad que requiere, en la mayoría de los casos, la interacción con los otros en torno a un tema o problema específico. Comprender la complejidad de la actividad argumentativa exige descentrarnos del sujeto y del argumento que él produce en un momento determinado. En términos de los autores, la argumentación es un tipo particular de diálogo que contribuye a que los individuos adquieran aprendizajes en temas específicos, así como en lo relacionado con prácticas culturales; los contextos socioculturales específicos en los cuales ocurre la argumentación orientan, limitan y contribuyen a la forma como se presente finalmente el argumento. En este sentido, la argumentación es siempre situada, se da siempre en un lugar y tiempo específico. Así mismo, nos permite comprender las características del interlocutor. En cuanto al tópico específico en el cual se realiza la actividad argumentativa es claro que este puede facilitar o limitar el proceso argumentativo, el cual también se ve afectado por las herramientas empleadas en la argumentación.

- En cuanto a los temas específicos, el desarrollo de habilidades argumentativas en el aula exige, de parte del maestro, la planeación detallada de los procesos de transposición didáctica. La interacción entre el proponente, el oponente y el objeto de discusión puede considerarse, para el caso de la argumentación, en un triángulo interactivo cercano al pro- puesto por Chevallard para la enseñanza de las ciencias (figura 3).

Figura 3. Argumentación como proceso de interacción entre el proponente, el oponente y el objeto de discusión en un contexto determinado

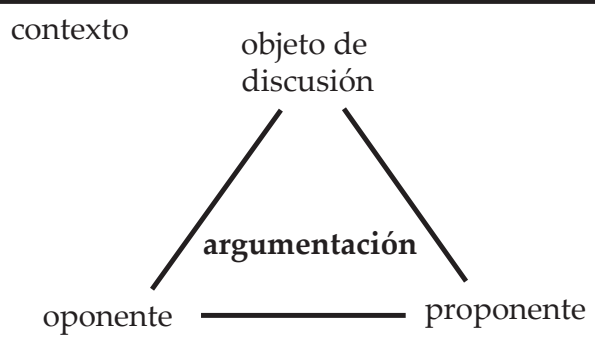

La interacción entre estos tres componentes exige de parte del maestro reflexiones en torno a las transformaciones permitidas entre el objeto de saber, el de enseñanza y el de aprendizaje. A su vez, este componente conceptual es sensible a aspectos ontológicos, cognitivo-lingüísticos y motivacionales de los participantes en el proceso argumentativo.

La experiencia de los sujetos, su aspecto ontológico, es determinante del desempeño argumentativo. La experiencia argumentativa, de discusión, de confrontación, en la que han participado las personas a lo largo de su vida, constituyen los presaberes o los modelos argumentativos que ponen en ejercicio los estudiantes en un momento determinado.

Vinculado con el aspecto ontológico, las habilidades cognitivo-lingüísticas de los estudiantes hacen referencia, de manera específica, a los usos del lenguaje y a aquellas habilidades cognitivas que ejercitan los estudiantes, tales como el análisis, la síntesis, la teorización y la conceptualización, entre otras, durante el proceso de argumentación. 
Además del lenguaje oral-escrito presente en las aulas de clase, encontramos que los procesos de enseñanza y aprendizaje, y con ellos el desarrollo de habilidades argumentativas, tienen como mediadores otros lenguajes (Lemke, 1999; Martins, 2000, 2001; Scott, 2000; Jewitt, 2000; Mortimer, 1998; Tamayo et ál., 2011). Se hace referencia, por ejemplo, al lenguaje oral, escrito, gestual, gráfico y corporal, entre otros.

Dentro de los aspectos cognitivos en el proceso de elaboración de argumentos uno es de especial importancia: el conocimiento y control que se tiene sobre los propios procesos del pensamiento, conocido como metacognición, determinante en el logro de aprendizajes profundos por parte de los estudiantes (Tamayo, 2006). El vínculo entre metacognición y argumentación es sugerente tanto en el ámbito eminentemente teórico como en el plano de la acción en las aulas de clase, de tal manera que regular de forma conciente e intencionada los desempeños argumentativos se constituye en uno de los fines de la enseñanza y el aprendizaje de las ciencias (Arenas, Sánchez y Tamayo, en prensa). Cuando un estudiante identifica sus fortalezas y debilidades al participar en espacios argumentativos $\mathrm{y}$, a su vez, tiene la capacidad para monitorear, evaluar y regular sus desempeños, tiene conciencia acerca de sus capacidades o competencias argumentativas. Este, sin duda, se constituye en uno de los propósitos centrales en la enseñanza.

La argumentación, como todo actuar de los seres humanos, es sensible a las motivaciones y a los afectos. De manera análoga a lo mencionado acerca de la metacognición, la metaafectividad se refiere al conocimiento y control de los propios procesos afectivos.
Conocer y regular nuestras expresiones de afectividad en el proceso de elaborar argumentos sin duda nos acerca a un mejor conocimiento de nuestra cognición y, de manera específica, de los procesos afectivos involucrados en el aprendizaje en dominios específicos del conocimiento.

\section{ReFERENCIAS}

Adam, J. M. (1995). Hacia una definición de la secuencia argumentativa. Comunicación, lenguaje y educación, 25, 9-22.

Bachelard, G. (1994). La formación del espíritu científico. México: Siglo XXI.

Bruner, J. (1987). La importancia de la educación. Barcelona: Paidós.

Campaner, G. y De Longhi, A. (2007). La argumentación en educación ambiental. Una estrategia didáctica para la escuela media. Revista Electrónica de Enseñanza de las Ciencias, 6 (2), 442-456.

Candela, A. (1999). Ciencia en el aula. Los alumnos entre la argumentación y el consenso. México: Paidós.

Caravita, S. y Hallden, O. (1994). Re-framing the problem of conceptual change. Learning and Instruction, 4, 89-111.

Cross, R. T. (1999). The public understanding of science: implications for education. International journal of science education, 7 (21), 699-702.

De Vos y Reiding. (1999). Public understanding of science as a separate subject in secundary school in the Netherlands. International journal of science education, 7 (21), 711-719.

Driver, R. y Newton, P. (1997). Establishing the norms of scientific argumentation inclassroom. Paper prepared for presentation at the ESERA conference. Roma. 
Duschl, R. A. y Gitomer, D. H. (1991). Epistemological perspectives on conceptual change: Implications for educational practice. Journal of Research in Science Teaching, 9 (28), 839-858.

Duschl, R. A., y Gitomer, D. H. (1997). Strategies and challenges to changing the focus of assessment and instruction in science classrooms. Educational Assessment, 1 (4), 37-73.

Duschl, R. A. y Osborne, J. (2002). Supporting and promoting Argumentation in Science Education. Studies in science Education, 38, 39-72.

Ennis, R. (1985). Critical thinking and the curriculum. National Forum, 65, 28-31.

Erdurán, S. (2008). Methodological foundations in the study of argumentation in science classroom. In: Jiménez-Alexandre y Erduran (Eds.) Argumentation in Science Education. Perspectives from classroom-based research (pp 47-69). USA: Springer.

Erdurán, S., Simon, S.y Osborne, J. (2004). Tapping into argumentation: Developments in the application of Toulmin's argument pattern for studying science discourse. Science Education. 6 (88), 915933.

Ericsson, K. A. and Kintsch, W. (1995). Long-term working memory. Psychological Review, 2, 211-245.

Facione, P. (2007). Pensamiento crítico: ¿qué es y por qué es importante? Chicago: Loyola University. Disponible en http://www. insightassessment.com

Fensham, P.J. y Harlem, W. (1999). School science and public understanding of science. International journal of science education, 7 (21), 755-763.
Giere, R. (1992). La explicación de la ciencia. Un acercamiento cognoscitivo. México: Consejo Nacional de Ciencia y Tecnología.

Guzmán, S. y Sánchez E., P. (2006). Efectos de un programa de capacitación de profesores en el desarrollo de habilidades de pensamiento crítico en estudiantes universitarios en el sureste de México. Revista Electrónica de Investigación Educativa, 2 (8). 12 de mayo 2006. Disponible en http://redie.uabc.mx/vol8no2/contenido-guzman.html

Jewitt, C. (2000). Formas de comunicación en la clase de ciencias. En Enseñar ciencias: construcción de significados i comunicación multimodal. Universidad de Barcelona. Documento no publicado.

Jiménez-Alexandre, M. P. (2008). Designing argumentation learning environment. In: Jiménez-Alexandre y Erduran (Eds.) Argumentation in Science Education. Perspectives from classroom-based research (pp. 91-115). USA: Springer.

Jiménez-Aleixandre, M. P. y Díaz de Bustamante, J. (2003). Discurso de aula y argumentación en la clase de ciencias: cuestiones teóricas y metodológicas. Enseñanza de las ciencias, 3 (21), 359-370.

Lemke, J.L. (1997). Aprender a hablar ciencia. Barcelona: Paidós.

Lemke, J.L. (1999). Meaning-making in the conversation: Head spinning, heart winning, and everything in between. Human Development, 42, 87-91.

Lipman, M. (1998). Pensamiento complejo y educación (2a․ ed.) Madrid: De la Torre.

Martins, I. (2000). Onda ou particula: Argumentaçao e robótica na aprendizagem da natureza da luz. Trabalho apresentado e publicaro nas Atas do VII Encontro de 
pesquisa em ensino de física. Florianópolis, SC, Brasil, 27-31 de mayo.

Martins, I. (2001). Anàlisi retórica i recerca en educació científica. Seminario dereserca. Universidad de Barcelona. Documento no publicado.

Mercer, N. (2009). Developing argumentation: Lessons learned in the primary school. In: Muller M. N. y Perret-Clermont, A-N. (Eds.). Argumentation and Education. Theoretical foundations and practice (pp. 177-194). Breinigsville: Springer.

Mockus, A. (1989). Formación básica y actitud científica. Educación y Cultura, 17.

Mortimer, E. F. (1998). Multivoicedness and univocality in classroom discourse: an example from the theory of matter. International Journal of Science Education, 20 (1), 67-82.

Muller, M. N., Perret-Clermont, A. N., Tartas, V. y Iannaccone, A.(2009). Psychosocial processes in Argumentation. In: Muller M. N. y Perret-Clermont, A. N. (Eds.). Argumentation and Education. Theoretical foundations and practice (pp. 67-90). Breinigsville, Springer.

Parodi, G. (2005). Discurso especializado e instituciones formadoras. Valparaíso: Ediciones Universitarias de Valparaíso.

Perelman, C. y Olbrechts-Tyteca, L. (1989). Tratado de la argumentación. La nueva retórica. Madrid: Gredos.

Puche, R. (2000). Formación de herramientas científicas en el niño pequeño. Universidad del Valle: Arango Editores.

Regh, W. (2010). Cogent Science in Context. The Science Wars, Argumentation Theory, and Habermas. Cambridge, MA: The MIT Press.
Revel Chion, A., Coulól, A., Erdurán, S., Furman, M., Iglesia, P. y Adúriz-Bravo, A. (2005). Estudios sobre la enseñanza de la argumentación científica escolar.

Riviére, A. (1986). Razonamiento y representación. Siglo XXI: España.

Sandoval, W. A. y Millwood, K. A. (2005). The Quality of Students' Use of Evidence in Written Scientific Explanations. Cognition and Instruction, 23 (1), 23-55.

Sandoval, W. A. (2001). Students' uses of data as evidence in scientific explanations. Paper presented at the Annual Meeting of the American Educational Research Assn. Seattle, WA, April 10-14.

Sardá, A. y Sanmartí, N. (2000). Enseñar a argumentar científicamente: un reto de las clases de ciencias. Enseñanza de las Ciencias, 3 (18), 405-422.

Sardá, A., Márquez, C. y Sanmartí, N. (2005). Cómo favorecer la comprensión de textos de ciencias. Enseñanza de las Ciencias, Número extra. VII Congreso.

Schwarz, B.B. (2009). Argumentation in Learning. In: Muller M. N. y PerretClermont, A-N. (Eds.). Argumentation and Education. Theoretical foundations and practice (pp. 91-126). Breinigsville: Springer.

Scott, P. (2000). Teacher talk and meaningmaking in science classrooms. En Enseñar ciencias: construcción de significados i comunicación multimodal. Barcelona: Universidad de Barcelona. Documento no publicado.

Spelke, E. (1991). Physical knowledge in infancy: Reflections on Piaget's theory, In: Carey, S. y Gelman, R. (Eds.). The epigenesis of mind: essays on biology and cognition. Erlbaum: Hillsdale, NY. 
Sutton, C. (1998a). Science as conversation: come and see my air pump! Wellington, J. (Ed.). Practical work in school science. Which way now? London: Routledge.

Sutton, C. (1998b). New perspectives on language in science. Fraser, B. J. y Tobin, K. G. (Eds.). International Handbook of Science Education, 27-38.

Tamayo, A. O. E. (2001). Evolución conceptual desde una perspectiva multidimensional. Aplicación al concepto de respiración. Tesis doctoral. Universidad Autónoma de Barcelona.

Tamayo, A. O. E. (2006). La metacognición en los modelos para la enseñanza y el aprendizaje de las ciencias. En Los bordes de la pedagogía: del modelo a la ruptura (pp. 275-306). Bogotá: Universidad Pedagógica Nacional.
Tamayo, A. O. E. (2009). Didáctica de las ciencias: La evolución conceptual en la enseñanza y el aprendizaje de las ciencias. Manizales: Editorial Universidad de Caldas.

Tamayo, A. O. E. y Restrepo, F. (2011). Niños y maestros. El caso de Pequeños Científicos. Manizales: Universidad Autónoma de Manizales.

Toulmin, S. (1977). La racionalidad humana. El uso colectivo y la evolución de los conceptos. Madrid: Alianza.

Toulmin, S. (2007). Los usos de la argumentación. Barcelona: Península.

Van Dijk, T. A. (1989). Handbook of discourse analysis. Great Britain: Academic press.

Zohar, A. (1999). Teachers metacognitive knowledge and the instruction of higher order thinking. Teaching and Teacher Education, 15, 413-429. 